\title{
Flat Waveguide with a Longitudinal Slot
}

\author{
Jan Zehentner, Jan Mrkvica, Jan Machac \\ Czech Technical University in Prague, Technicka 2, 16627 Prague, Czech Republic
}

\begin{abstract}
This paper presents an analysis of a flat slotted waveguide by the spectral domain method finalized by a complex root search procedure. Matching of fields from bounded and unbounded regions at the common interface is explained and applied to satisfy the boundary conditions in the spectral domain. New theoretical findings are reported, and the dispersion characteristics of particular modes are shown and discussed with regard to their potential application. The different behaviour of the odd and even modes is pointed out. Their affinity to the modes in a standard rectangular waveguide is noted. A hint is given of a modification of the line cross-section resulting in the propagation of a bound mode besides the propagation of the space leaky modes.
\end{abstract}

Index Terms - Boundary conditions, complex propagation constants, flat slotted waveguide, leaky waves, spectral domain method.

\section{INTRODUCTION}

Printed circuits are very popular and have extensive applications in $\mathrm{mw}$ and $\mathrm{mmw}$ technology. Planar transmission lines are a significant integral part of printed circuits. Many planar transmission lines have therefore been thoroughly analyzed for a long time. Planar or uniplanar lines are mostly open, being a counterpart to closed, or shielded lines. Open transmission lines leak power in some frequency band. We successively investigated the slotline, the conductor-backed slotline, the inverted conductor-backed slotline, the coplanar waveguide and the conductor-backed coplanar waveguide in order to find a line leaking power only in the half-space. However, all of them exhibit not only the required leakage into the space, but also undesirable leakage into the substrate. Multilayered or stacked substrates and vias for suppressing leakage into the substrate are less well fabricated in practice. Based on our experiments and our theoretical analysis of many planar low profile transmission lines we concluded that a transmission line suitable for the design of a planar leaky wave antenna must have a substrate of finite width and thickness, shielded on the side and bottom walls and carrying a longitudinal slot on the top. Low cross-section height is an important feature determining the method of analysis.

On the other hand, traveling wave slot antennas made of waveguides were designed, investigated and measured fifty years ago [1]-[2]. However, the concept of their analysis was based on methods used in classic waveguide techniques. The height of the waveguide was greater, comparable with its width. Our approach removes this limitation and is valid for both low and high profile transmission lines.
This paper presents an analysis of a flat waveguide with a longitudinal slot, reports new theoretical findings, illustrates characteristics of the line and predicts its applicability. The line is analyzed by the spectral domain approach with a complex root searching procedure.

\section{DISPERSION CHARACTERISTICS OF THE LINE}

A conductor-backed slotline with a finitely wide substrate metallized also on its side walls is the subject of our interest. The cross-section of the line with its proportions and co-ordinates is shown in Fig. 1. Generally, $\varepsilon_{\mathrm{r} 1} \neq \varepsilon_{\mathrm{r} 2}$ and $\varepsilon_{\mathrm{r} 2}>1$. When $b$ is substantially greater than $h$ we have a flat waveguide with a slot cut in the waveguide wall parallel to its longitudinal axis. In order to judge the line behaviour, its complete dispersion characteristics are needed.

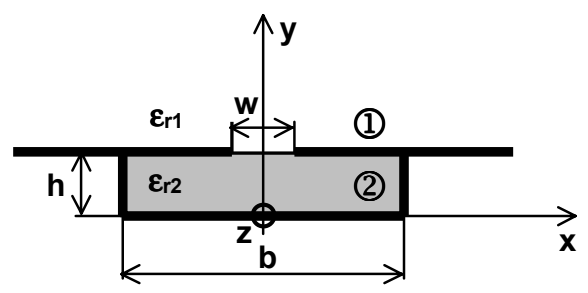

Fig. 1 Cross-section of a flat slotted waveguide

We analyzed the line by the method of moments in the spectral domain applying Galerkin's procedure terminated by complex root searching. We assumed zero thickness of the top metallization, a lossless line, its uniformity in the $z$-direction and $\exp \left(-j \gamma_{z} z\right)$ dependence. A specific aspect of this analysis is matching of the fields at the dielectric interface in the slot. The field is represented by the longitudinal components of the electric and magnetic Hertz potentials $\Pi^{e}=\mathbf{z}_{0} \Pi^{e}$ and $\Pi^{m}=\mathbf{z}_{0} \Pi^{m}$. The Fourier transform of $\Pi^{e, m}$ in the upper boundless region, when indices $e, m$ are omitted, is

$$
\widetilde{\Pi}_{1}(\xi, y)=\int_{-\infty}^{\infty} \Pi_{1}(x, y) \mathrm{e}^{-j \xi x} d x .
$$

In the second region, laterally bound, the integral taken over the finite interval $b$ defines the Fourier transform

$$
\widetilde{\Pi}_{2}\left(\xi_{n}, y\right)=\frac{2}{b} \int_{-b / 2}^{b / 2} \Pi_{2}(x, y) \mathrm{e}^{-j \xi_{n} x} d x,
$$

where 


$$
\xi_{n}= \begin{cases}\frac{2 n \pi}{b} & \text { for even modes } \\ \frac{(2 n-1) \pi}{b} & \text { for odd modes }\end{cases}
$$

The modes may have even or odd symmetry of the electric field component $E_{x}$ parallel to the interface in the slot $y=h$ with regard to the plane of the line symmetry $x=0$. The solution of the wave equation

$$
\left[\frac{\partial^{2}}{\partial y^{2}}-\gamma_{1,2}^{2}\right] \widetilde{\Pi}_{1,2}=0
$$

in the spectral domain $\widetilde{\Pi}_{1}(\xi, y)$ or $\widetilde{\Pi}_{2}\left(\xi_{n}, y\right) y$-dependent is known and

$$
\begin{aligned}
& \gamma_{1}^{2}=\xi^{2}+\gamma_{z}^{2}-\varepsilon_{r 1} k_{0}^{2} \\
& \gamma_{2}^{2}=\xi_{n}^{2}+\gamma_{z}^{2}-\varepsilon_{r 2} k_{0}^{2},
\end{aligned}
$$

where $\gamma_{1,2}$ are separation constants in the 1 st and 2 nd area.

In the 1st region we have two and in the 2nd region four unknown integration constants. To determine them, the boundary conditions are imposed at the tangential components of the electric and magnetic field on the plane $y=h$ and transformed to the spectral domain. The fields are expressed in terms of the Fourier integral, by analogy with (1) and (2), taken in the boundless and bound intervals in the 1st and 2nd region, respectively. Consequently, the relation between these Fourier transforms mediated by the so called finite function are [3]

$$
\begin{aligned}
& \widetilde{E}_{x 1}(\xi, h)=\frac{b}{2} \sum_{n=-\infty}^{\infty} \widetilde{E}_{x 2}\left(\xi_{n}, h\right) \cdot \mathrm{Sa}\left[\frac{b}{2}\left(\xi-\xi_{n}\right)\right] \\
& \widetilde{E}_{z 1}(\xi, h)=\frac{b}{2} \sum_{n=-\infty}^{\infty} \widetilde{E}_{z 2}\left(\xi_{n}, h\right) \cdot \mathrm{Sa}\left[\frac{b}{2}\left(\xi-\xi_{n}\right)\right] \\
& \widetilde{J}_{x}(\xi, h)=\widetilde{H}_{z 1}(\xi, h)-\frac{b}{2} \sum_{n=-\infty}^{\infty} \widetilde{H}_{z 2}\left(\xi_{n}, h\right) \cdot \mathrm{Sa}\left[\frac{b}{2}\left(\xi-\xi_{n}\right)\right] \\
& \widetilde{J}_{z}(\xi, h)=-\widetilde{H}_{x 1}(\xi, h)+\frac{b}{2} \sum_{n=-\infty}^{\infty} \widetilde{H}_{x 2}\left(\xi_{n}, h\right) \cdot \mathrm{Sa}\left[\frac{b}{2}\left(\xi-\xi_{n}\right)\right]
\end{aligned}
$$

where the sample function

$$
\mathrm{Sa}\left[\frac{b}{2}\left(\xi-\xi_{n}\right)\right]=\frac{\sin \left[\frac{b}{2}\left(\xi-\xi_{n}\right)\right]}{\frac{b}{2}\left(\xi-\xi_{n}\right)} .
$$

$E_{x}(x, h)=e_{x}(x, h), E_{z}(x, h)=e_{z}(x, h)$ are hitherto unknown electric field distributions nonzero only within the slot. Let $e_{x}(x, h)$, $e_{z}(x, h)$ be represented in terms of the series of basis functions with unknown amplitudes $c_{x}, c_{z}$ and $d_{x}, d_{z}$. After their transformation to the spectral domain

$$
\begin{aligned}
& \widetilde{E}_{x 1}(\xi, h)=\widetilde{e}_{x}(\xi, h) \\
& \widetilde{E}_{z 1}(\xi, h)=\widetilde{e}_{z}(\xi, h) \\
& \widetilde{E}_{x 2}\left(\xi_{n}, h\right)=\widetilde{e}_{x}\left(\xi_{n}, h\right) \\
& \widetilde{E}_{z 2}\left(\xi_{n}, h\right)=\widetilde{e}_{z}\left(\xi_{n}, h\right)
\end{aligned}
$$

where

$$
\begin{aligned}
& \widetilde{e}_{x}(\xi, h)=\sum_{i=1}^{\infty} c_{x i} \widetilde{e}_{x i}(\xi) \\
& \widetilde{e}_{z}(\xi, h)=\sum_{j=1}^{\infty} c_{z j} \widetilde{e}_{z j}(\xi) \\
& \widetilde{e}_{x}(\xi, h)=\sum_{k=1}^{\infty} d_{x k} \widetilde{e}_{x k}\left(\xi_{n}\right) \\
& \widetilde{e}_{z}\left(\xi_{n}, h\right)=\sum_{l=1}^{\infty} d_{z l} \widetilde{e}_{z l}\left(\xi_{n}\right)
\end{aligned}
$$

and the basis functions $e_{x i}, e_{z j}$ for even and odd modes are cos and $\sin$ functions modified by the rapidly growing term accounting for the field at the slot edges [3]. They hold for $|x| \leq w / 2$, and outside this interval they are zero. Successive substitution of (12) into (11) and further into (6)-(9) results in a set of equations relating unknown current densities $\widetilde{J}_{x}(\xi, h)$, $\widetilde{J}_{z}(\xi, h)$ on the metallization and unknown electric fields represented by $\widetilde{e}_{x}(\xi, h), \widetilde{e}_{z}(\xi, h), \widetilde{e}_{x}\left(\xi_{n}, h\right), \widetilde{e}_{z}\left(\xi_{n}, h\right)$ in the slot. The currents and electric fields are nonzero in the complementary region of the $y=h$ plane. Consequently, application of Parseval's theorem excludes currents from these equations. Galerkin's method was used for solving the set of equations for amplitudes of the basis functions $c_{x i}, c_{z j}, d_{x k}, d_{z l}$. Its nontrivial solution is obtained when the determinant of the set of equations equals zero. This condition is the well known dispersion equation, which is fulfilled for the propagation constant of the searched mode.

The electric field in the 1 st region follows from the backward Fourier transform

$$
E_{1 p}(x, y)=\frac{1}{2 \pi} \int_{-\infty}^{\infty} \widetilde{E}_{1 p}(\xi, y) e^{j \xi x} d \xi
$$

and by analogy in the 2 nd region

$$
E_{2 p}(x, y)=\frac{1}{2} \sum_{n=-\infty}^{\infty} \widetilde{E}_{2 p}\left(\xi_{n}, y\right) e^{j \xi_{n} x} d \xi
$$

where $p=x, y, z . \widetilde{E}_{1 p}(\xi, y)$ and $\widetilde{E}_{2 p}\left(\xi_{n}, y\right)$ are now determined by (11), where the amplitudes of the basis functions are known from the set of homogeneous equations leading to the propagation constant search.

If needed, the characteristic impedance can be calculated according to the power-voltage definition. The voltage across the slot is computed in the space domain, while the transmitted power is calculated more easily in the spectral domain, provided that Parseval's theorem is used. The definition of the characteristic impedance is not applicable to odd modes since the voltage across the slot is zero. 


\section{DISCUSSION OF RESULTS}

The complex propagation constant on a transmission line with a slot in the wider wall of an air-filled waveguide with $\mathrm{TM}_{11}$ excitation was measured and calculated in [1]. It was also calculated by a transverse resonant procedure employing a transverse equivalent network and the perturbation solution of the resonance equation [2]. A code for computing the complex propagation constant by the method presented in this paper has been written and tested. Comparisons of data from [1] and [2] with our own data for the squared slotted waveguide are shown in Figs. 2 and 3 where $h=b$. The agreement is acceptable. We therefore conclude that the code is correct and reliable.

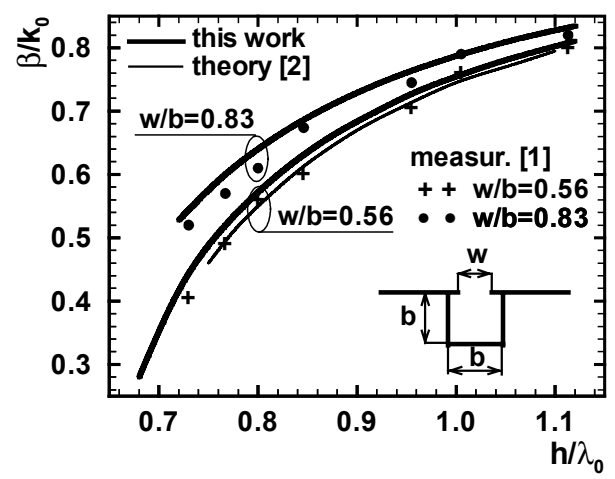

Fig. 2 Normalized phase constant of the squared slotted waveguide

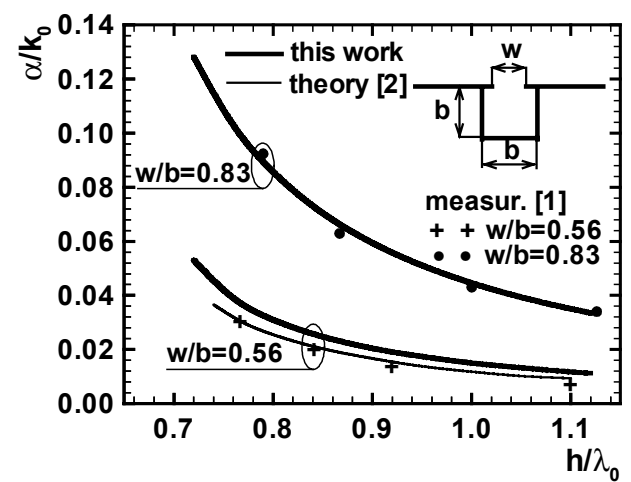

Fig. 3 Normalized leakage constant of the squared slotted waveguide

Let us observe the first odd space leaky modes. The phase and leakage constants of the first five modes, when $\varepsilon_{r l}=\varepsilon_{r 2}=1$, related to $\mathrm{k}_{0}$ are shown in Fig. 4. These modes correspond to modes of the rectangular waveguide perturbed by the longitudinal slot. We distinguish them by the order number. The first space leaky mode is related to the dominant mode of the waveguide $\mathrm{TE}_{10}$. The second mode corresponds to the $\mathrm{TM}_{11}$ mode, and the field of the third mode resembles the field of the $\mathrm{TE}_{11}$ mode. The fourth space leaky mode is a modification of the $\mathrm{TM}_{12}$ mode, while the fifth has a link to the $\mathrm{TE}_{12}$ mode. The characteristics of the $2 \mathrm{nd}$ and $3 \mathrm{rd}$, and also the 4th and 5th modes, are nearly superimposed on each other in Fig. 4 . The phase constants of particular modes trace $\beta$ in the waveguide above their cut-offs according to

$$
\beta-j \alpha=\sqrt{k_{0}^{2}-\left(\frac{m \pi}{b}\right)^{2}-\left(\frac{n \pi}{h}\right)^{2}}
$$

and are marked out by dots in Fig. 4. Similarly, the attenuation constant $\alpha$, typical for evanescent modes below the cut-offs, coincides with the space leakage constants. Accordingly, a waveguide with a narrow slot does not radiate since the leakage constant is very great below the cut-off of the waveguide, as is confirmed by each measuring slotted line. Similarly it does not radiate above the cut-off, since $\alpha$ is almost zero.

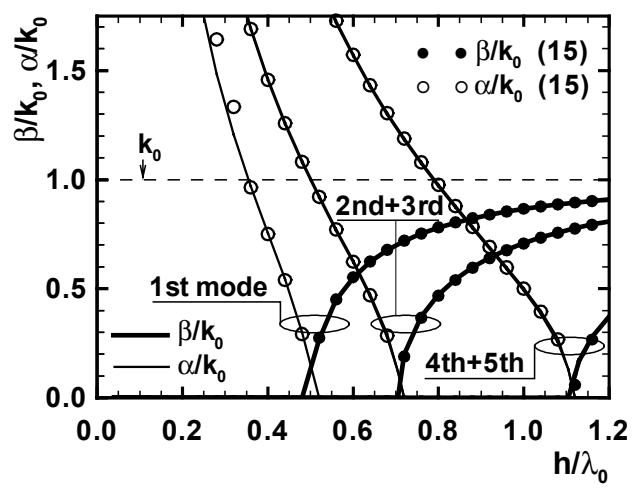

Fig. 4 Normalized dispersion characteristics of five first odd space leaky modes on the squared slotted waveguide with $\mathrm{w}=1 \mathrm{~mm}$, $\mathrm{h}=\mathrm{b}=10 \mathrm{~mm}, \varepsilon_{\mathrm{r} 1}=\varepsilon_{\mathrm{r} 2}=1$

The field distribution inside the waveguide influences the field in the space and correspondingly changes the propagation constant. For a wide slot the dispersion characteristics of the 2nd and 3rd mode, and also of the 4th and 5th mode, become separated. The narrower the slot-width, the closer together are the frequencies at which $\beta$ and $\alpha$ approach zero. This effect is shown in Fig. 5, where the influence of the $\varepsilon_{r 2}$ permittivity in comparison to Fig. 4 is also documented. Greater substrate permittivity shifts the characteristics to lower frequencies. Note that the space leaky mode converts into a bound mode when $\beta=\mathrm{k}_{0}$.

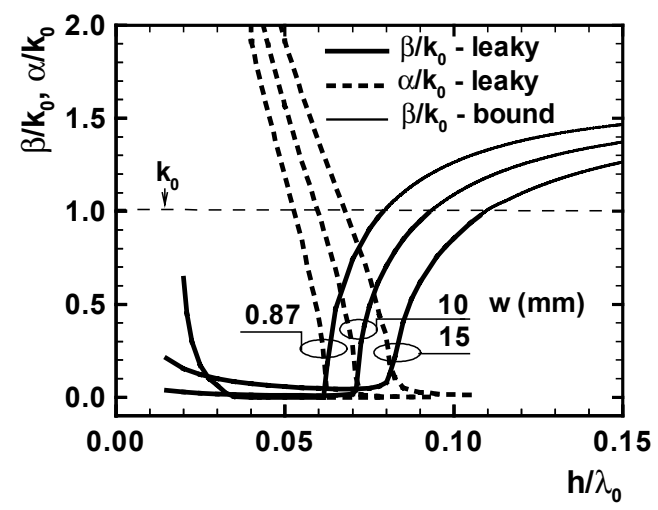

Fig. 5 Normalized dispersion characteristics of the first odd space leaky mode on the flat slotted waveguide when $\mathrm{w}=0.87,10.0,15.0$ $\mathrm{mm}, \mathrm{b}=30 \mathrm{~mm}, \mathrm{~h}=6 \mathrm{~mm}, \varepsilon_{\mathrm{r} 2}=2.6, \varepsilon_{\mathrm{r} 1}=1$ 
Now let us note the even modes on the completely shielded conductor-backed slotline. The normalized dispersion characteristics of the first two modes are plotted in Fig. 6. The conversion of the space leaky mode into the bound mode setting up at the frequency when the phase constant crosses one is again evident. The space leaky mode propagates below this frequency, while the bound mode propagates above it. It turned out that when $\varepsilon_{r l}=\varepsilon_{r 2}$ the odd and even bound modes cannot propagate, only space leaky modes are allowed. However, when $\varepsilon_{r 2}>\varepsilon_{r l}$ the space leaky modes convert at higher frequencies into the bound modes, as is shown in Figs. 5 and 6 . Owing to lack of space, the transversal component $E_{T}(x, y)$ of the space leaky modes is not here given.

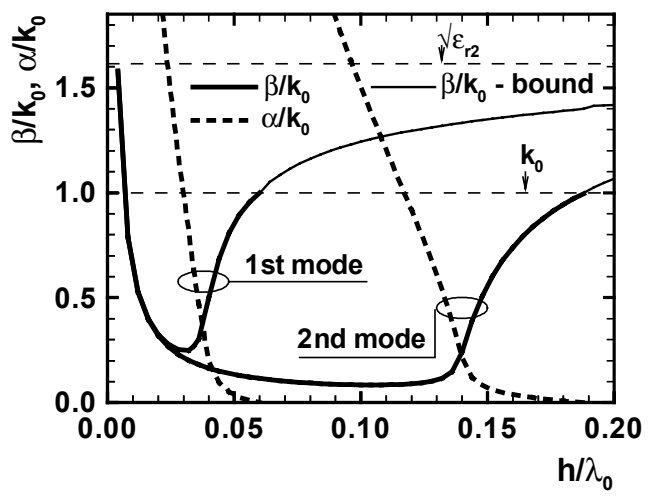

Fig. 6 Normalized dispersion characteristics of the first two even space leaky modes on the flat slotted waveguide when $\mathrm{w}=2 \mathrm{~mm}$, $\mathrm{b}=30 \mathrm{~mm}, \mathrm{~h}=6 \mathrm{~mm}, \varepsilon_{\mathrm{r} 2}=2.6, \varepsilon_{\mathrm{r} 1}=1$

Discussion about a modal spectrum can be supplemented by the following modification of the line. A dielectric slab with permittivity $\varepsilon_{r}>1$ and thickness $h_{s}$ placed on the upper metallization of the flat waveguide $\left(\varepsilon_{r l}=\varepsilon_{r 2}=1\right)$ totally changes the dispersion characteristics of the even modes, as is shown in Fig. 7. When $\beta$ becomes $k_{0}$, the space leaky mode converts into the bound mode which propagates up to the frequency when $\beta=k_{T M 0}$. Then a spectral gap occurs. Above its upper limit, when again $\beta=k_{T M 0}$, leakage into the dielectric slab sets up.

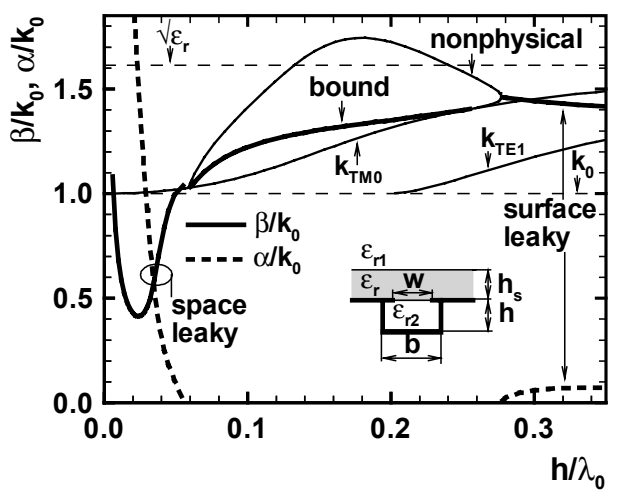

Fig. 7 Normalized dispersion characteristics of even modes on the flat slotted waveguide when $\mathrm{w}=2 \mathrm{~mm}, \mathrm{~b}=30 \mathrm{~mm}, \mathrm{~h}=\mathrm{h}_{\mathrm{s}}=6 \mathrm{~mm}, \varepsilon_{\mathrm{r}}=2.6$, $\varepsilon_{\mathrm{r} 2}=\varepsilon_{\mathrm{r} 1}=1$

\section{CONCLUSION}

This paper has presented an analysis of a waveguide with a longitudinal slot cut in the middle of the wider wall, extended laterally to infinity. The analysis used the method of moments in the spectral domain. The crucial point was the formulation of the boundary conditions in the spectral domain on the plane separating the boundless half-space and the bound volume of the substrate. In other words, this involved matching the Fourier integral and Fourier series in the space domain. In the end, the standard Galerkin method and the complex root searching technique were applied.

Two groups of modes, odd and even, related to the odd and even symmetry of the transversal component of the electric field in the slot, were identified and can propagate on the line. Odd space leaky modes are accessible in the flat slotted waveguide. Their series is related to the series of modes existing in the rectangular waveguide perturbed by the slot. The growth of the slot-width separates their propagation constants from each other. The permittivity of the substrate moves the dispersion characteristics towards lower frequencies. Space odd modes are perturbed waveguide modes, while space even modes are perturbed slot-type modes. When the permittivity of the substrate in the waveguide is greater than permittivity outside, the space leaky modes convert into the bound modes. The conversion occurs when $\beta$ crosses $\mathrm{k}_{0}$. A dielectric slab covering the upper surface of the line narrows the frequency band of the space leakage, allows propagation of the bound mode and permits leakage into that slab above the spectral gap.

This work, motivated by an investigation of the space leaky modes on a conductor-backed slotline with a finite substrate width, is an alternative analysis of the slotted rectangular waveguide. It gives a better understanding of the guided wave phenomenon, clarifies its physical background and provides subsequent practical applications. It does not impose any restriction on the line proportions.

\section{ACKNOWLEDGEMENT}

This work has been supported by the Grant Agency of the Czech Republic under project 102/03/0449 "New circuit devices for communication technology".

\section{REFERENCES}

[1] J. N. Hines, V. H. Rumsey and C. H. Walter, "Traveling-Wave Slot Antennas," Proc. IRE, vol. 41, pp. 1624-1631, November 1953.

[2] L. O. Goldstone and A. A. Oliner, "Leaky-Wave Antennas I: Rectangular Waveguides," IRE Trans. Antennas \& Propagation, vol. 7, pp. 307-319, October 1959.

[3] J. Mrkvica, Wave propagation on modified slotlines and coplanar lines, $\mathrm{PhD}$ Thesis (in Czech), Prague: CTU in Prague, 2004. 